Bicocca-FT/00/05,

hep-th/0004070

\title{
type IIB orbifolds with D5-branes and their string duals ${ }^{\dagger}$
}

\author{
Andrea Gregori ${ }^{1}$ \\ Dipartimento di Fisica, Università di Milano-Bicocca \\ and \\ INFN, Sezione di Milano, Italy
}

\begin{abstract}
We consider $Z_{2}$, freely acting orbifolds of the type IIB string with 16 parallel D5-branes. When the string is compactified on $T^{2} \times T^{4}$ and the D5-branes are wrapped on $T^{2}$, these systems possess $\mathcal{N}_{4}=2$ supersymmetry, originating from the spontaneous, partial breaking of $\mathcal{N}_{4}=4$. Extended supersymmetry allows us to investigate the duality with certain heterotic and type I constructions, and to obtain informations about their non-perturbative regime.
\end{abstract}

${ }^{\dagger}$ Research partially supported by the EEC under the contract TMR-ERBFMRX-CT96-0045.

${ }^{1}$ e-mail: agregori@pcteor.mi.infn.it 


\section{Introduction}

Systems of type IIB strings in the presence of $N$ parallel Dp-branes are of much interest for the investigation of the non-perturbative aspects of Yang-Mills theories. A well studied situation is when one takes the limit of large $N$, small $g_{s}$. In this limit, gravity is effectively decoupled, and one reduces to study a pure gauge theory. Recently, situations in which the string coordinates transverse to the D-branes enter in the game have also been considered [1]. In this work, we will only consider BPS D-branes; the presence of such D-branes leads to the breaking of half of the supersymmetries of the type IIB string. When the four coordinates transverse to the branes are toroidally compactified, cancellation of the Ramond-Ramond charge requires the introduction of orientifold O5 planes and fixes the number of D5-branes to be sixteen, toghether with their mirror pairs. This construction can be seen as an orientifold of the type IIB string, projected by $\tilde{\Omega} \equiv \Omega \times I_{(7,8,9,10)}$, the product of the world-sheet parity times the target-space reflection acting on the transverse coordinates. This orbifold is therefore related to the ordinary orientifolds by T-duality along $x_{7}, x_{8}, x_{9}, x_{10}$, and it is reasonable to ask wether this theory is dual to the toroidally compactified heterotic string. The point is non trivial, because, on the type IIB string, supersymmetry appears as "spontaneously" broken, in the sense that the theory possesses an approximate restoration of the initial 32 supersymmetry charges when there is a local cancellation of the RamondRamond charge and the space transverse to the branes is decompactified. On the other hand, it is known that on the heterotic side, even at the strong coupling there are no more than 16 supercharges: the strong coupling limit is in fact the type IIA string compactified on K3. In the case of heterotic compactifications with maximal supersymmetry, such a duality can therefore exist only in a particular limit in the moduli space of the two theories. It is however possible that it exists for theories with a lower number of supersymmetries, in which the projection that breaks supersymmetry has a non-trivial action on the dilaton field. In order to understand the situation, we compactify two further coordinates on $T^{2}$ and consider the theory obtained by adding a freely acting, $Z_{2}$ projection that acts as a twist on the transverse coordinates and as a translation along a circle of the two-torus parallel to the branes. This projection breaks spontaneously the supersymmetry to $\mathcal{N}_{4}=2$ : the $\mathcal{N}_{4}=2$ and $\mathcal{N}_{4}=4$ phases are then continuosly related. In the $\mathcal{N}_{4}=2$ phase of the theory, the moduli dependence of certain amplitudes is reacher than in the $\mathcal{N}_{4}=4$ phase. Supersymmetry is nevertheless still sufficiently extended to allow the comparison of amplitudes that, like the $R^{2}$ term we will consider, receive a contribution only from non-perturbatively stable, BPS states.

When $V_{(4)}$, the volume of the space transverse to the D5-branes, is small, the ordinary type I orientifold description is indeed the more appropriate. The description in terms of D5branes turns out to be useful in order to investigate the limit $V_{(4)} \rightarrow \infty$, non-perturbative from the type I point of view. As we will discuss, in this limit there is an approximate restoration of a larger amount of supersymmetry. Due to the free action of the orbifold projection, in this model indeed act both a perturbative and a non-perturbative super-Higgs mechanism, responsible for the spontaneous breaking of the $\mathcal{N}_{4}=8$ supersymmetry. We argue that this model, T-dual to a type I freely acting orbifold with sixteen D9-branes, presented in Ref. [2], is also dual to a freely acting orbifold of the heterotic string, with 
gauge group of maximal rank. In order to substantiate our arguments, we consider the renormalization of the effective coupling of the $R^{2}$ term. We compare this quantity, as computed on the heterotic side, on the type I and in the "type IIB", $\tilde{\Omega}$ dual orientifold, both for $V_{(4)} \rightarrow 0$ and $V_{(4)} \rightarrow \infty$. Since the effective coupling of this term depends on the moduli of the vector multiplets, this comparison provides us with informations about the non-perturbative behavior of the theory.

The paper is organized as follows:

In Section 2 we discuss the type IIB orientifold with D5-branes, and the further breaking of supersymmetry produced by the orbifold projection. The main part of the section is devoted to the discussion of the gravitational corrections.

In Section 3 we present the heterotic and type I duals. We compare then the gravitational corrections as computed in all the constructions and discuss the map relating the moduli in the vector manifolds of the effective theories.

In Section 4 we review the procedure of rank reduction in type I strings. This was first discussed in [3]- 6], and we apply it to the case of type I models with spontaneous breaking of supersymmetry. We use then the results of the previous sections in order to infer the non-perturbative behavior of these constructions.

Further comments and conclusions are in Section 5.

\section{The type IIB string with D5-branes}

We start by considering the addition of parallel D5-branes to the type IIB superstring. When the space transverse to the D5-branes is compactified on $T^{4}$, the Ramond-Ramond charge of the branes must be cancelled. The cancellation is obtained by introducing orientifold O5 planes. By a chain of T-dualities applied to the O9 case, one can see that their number is fixed to sixteen: the allowed number of D5-branes is therefore also sixteen (toghether with their sixteen mirrors). If each mirror pair sits on one orientifold plane, the cancellation of the RR charge is local. This construction can be seen as an orientifold obtained by projecting the type IIB string with $\tilde{\Omega}$, defined as the product $\Omega \times I_{(7,8,9,10)}$, of the world-sheet parity, $\Omega$, and the target-space reflection $I_{x}: x \rightarrow-x$, along the four transverse coordinates. Tduality on the type IIB string, along these coordinates, relates therefore this theory to the ordinary type I, orientifold construction, in which the D9-branes and one O9 plane replace the D5-branes and the O5-planes. When the volume, $V_{(4)}$, of the space transverse to the D5-branes, is small, the type I orientifold indeed gives the more appropriate description of the theory, that corresponds to the phase in which the D-branes gauge group is broken to $U(1)^{16}$. The opposite limit, $V_{(4)} \rightarrow \infty$, is non-perturbative from the point of view of the effective theory. On the other hand, a simple look at the orientifold construction indicates that, since the $\tilde{\Omega}$ projection breaks T-duality along $T^{4}$, this limit is indeed very different from the other one: in this limit the bulk theory feels the D5-branes very weakly, and we expect that, being the supersymmetry breaking projection associated to the breaking of T-duality, to recover an approximate restoration of the original amount of supersymmetry, that appears therefore spontaneously broken for any finite value of $V_{(4)}$. It is not difficult to 
write an explicit expression for the partition function of this kind of orientifold: it can be obtained from the well known orientifold partition function with D5-branes, by performing a translation along the four circles transverse to the branes, up to the center of the segments connecting the two fixed points of each circle. In the closed string sector, the Torus, being invariant under translations, is not affected. The Klein bottle and the open string diagrams are instead affected; in these sectors the translation amounts to a half-integer shift of the winding numbers corresponding to the transverse coordinates. When the radii are small $\left(V_{(4)} \rightarrow 0\right)$, the theory recovers the ordinary, "unshifted" orientifold. In the large radii limit instead $\left(V_{(4)} \rightarrow \infty\right)$, the diagrams generated by the orientifold projection vanish, leaving only the type IIB partition function.

We now go to four dimensions by compactifying two other coordinates on a two-torus, and break the $\mathcal{N}_{4}=4$ supersymmetry with a freely acting, $Z_{2}$ orbifold projection, that acts as a twist, $x \rightarrow-x$, on $x_{7}, x_{8}, x_{9}, x_{10}$, and as a half circumference translation in a circle of $T^{2}$. This produces a spontaneous breaking of supersymmetry to $\mathcal{N}_{4}=2$ [7], due to a stringy version of the mechanism of Ref. [8]. This model is related by T-duality along $T^{4}$ to the "Scherk-Schwarz breaking" type I construction presented in Ref. [2]. The massless spectrum is therefore the same. Here however, having a local cancellation of the RamondRamond charge, necessary for the existence of a regular decompactification limit, requires to put each D5-branes pair on its corresponding orientifold plane. This leads to the breaking of the gauge group to $U(1)^{16}$. This configuration corresponds to introducing appropriate Wilson lines on the dual construction with D9-branes. Under T-duality, Wilson lines are in fact mapped into the positions of the D5-branes.

\section{The $R^{2}$ corrections}

We come to the computation of the corrections to the effective coupling of the $R^{2}$ term円. We are interested in the expression of the effective coupling in the two limits of large and small $V_{(4)}$. As we observed, for small $V_{(4)}$, the theory is perturbatively equivalent to a type I orientifold, with D9-branes instead of D5-branes. The computation of the gravitational corrections is therefore absolutely equivalent, modulo a redefinition of the fields in the vector manifold, due to the D5-/ D9-branes exchange, to the one already performed in that framework. Taking this into account, we can immediately write the correction to the effective coupling by appropriately adapting the result obtained for that model in Ref. [10] (see also Section 3):

$$
\frac{16 \pi^{2}}{g_{\text {grav }}^{2}(\mu)}=16 \pi^{2} \operatorname{Im} S^{\prime}-\frac{2}{3} \log \operatorname{Im} U\left|\vartheta_{4}(U)\right|^{4}-b \log \mu / M,
$$

where $U$ is the modulus associated to the complex structure of the unwisted two-torus and now $\left\langle\operatorname{Im} S^{\prime}\right\rangle$ is the tree level coupling of the D5-branes [1], expressed as the vacuum expectation value of the field $S^{\prime}$, defined as in Ref. [12 (see also Eq. (3.11) below). 2/3 is the value of the beta-function coefficient, $b_{\text {grav }}=\frac{24-N_{V}+N_{H}}{12}$. For the particular parametrization

\footnotetext{
${ }^{1}$ For a precise definition and a discussion of this amplitude in our notation, we refer the reader to [9].
} 
of the untwisted two-torus in which

$$
\operatorname{Im} T=R_{5} R_{6}, \quad \operatorname{Im} U=R_{6} / R_{5},
$$

the $\vartheta_{4}(U)$ appears when the orbifold projection that twists the four coordinates transverse to the branes acts on the two-torus as $(-1)^{m}$, on the momenta on $x_{6}$. The third term on the r.h.s. contains the infrared running, as a function of the infrared cut-off $\mu$ and the characteristic mass scale $M$. The ratio of these quantities is invariant under type II/heterotic/type I, string-string dualities, and can be expressed as the ratio of a physical cut off $\sqrt{p^{2}}$ and the Planck mass $M_{P}$. The $\beta$-function coefficient $b$, in the cases it can be explicitly computed (see for instance [13]), turns out to be $b=\frac{B_{4}-B_{2}}{3}$, with $B_{2}$ and $B_{4}$ the constant, massless contributions at a generic point in the moduli space (i.e. away from points of enhanced symmetry) to the second and fourth helicity supertraces (for a discussion of these quantities, see for instance [14]).

We consider now the limit of (very) large $V_{(4)}$. As we noticed, in the absence of the orbifold projection this would be a limit of approximate restoration of the full supersymmetry. In the present case, this is still true, provided the circle of the two-torus translated by the orbifold projection is appropriately decompactified, in order to make vanishing the orbifold action. In this limit, the correction to the gravitational coupling is expected to vanish (or, more generally, to diverge only as a logarithm of the moduli). For finite values of the radii of the two-torus, supersymmetry is instead broken to $\mathcal{N}_{4}=2$. The contribution of the D5-branes is on the other hand suppressed, and we expect that there is no tree level contribution to the correction. The full result comes therefore from one loop (owing to extended supersymmetry, there are no contributions from higher orders). The correction to the $R^{2}$ term is then well approximated by the expression obtained by inserting the "helicity operator" $Q^{2} \bar{Q}^{2}$, in the type IIB partition function. $Q$ and $\bar{Q}$ are the operators corresponding to the left and right helicity, and act as derivatives on the appropriately deformed partition function 2 . The gravitational amplitude is in fact proportional to an index (see Refs. [15, 16]) and, as shown in Refs. [9, 17], this insertion leads to the computation of the appropriate amplitude, provided one keeps the only $U$-dependent part of the result. The partition function we use for the one-loop computation is therefore:

$$
\begin{aligned}
Z_{\mathrm{bulk}}= & \frac{1}{\operatorname{Im} \tau|\eta|^{20}} \frac{1}{2} \sum_{\alpha, \beta}(-1)^{\alpha+\beta+\alpha \beta} \theta^{2}\left[\begin{array}{c}
\alpha \\
\beta
\end{array}\right] \frac{1}{2} \sum_{\bar{\alpha}, \bar{\beta}}(-1)^{\bar{\alpha}+\bar{\beta}+\bar{\alpha} \bar{\beta}} \bar{\theta}^{2}\left[\begin{array}{c}
\bar{\alpha} \\
\bar{\beta}
\end{array}\right] \\
& \times \frac{1}{2} \sum_{H, G} \theta\left[\begin{array}{c}
\alpha+H \\
\beta+G
\end{array}\right] \theta\left[\begin{array}{c}
\alpha-H \\
\beta-G
\end{array}\right] \bar{\theta}\left[\begin{array}{c}
\bar{\alpha}+H \\
\bar{\beta}+G
\end{array}\right] \bar{\theta}\left[\begin{array}{c}
\bar{\alpha}-H \\
\bar{\beta}-G
\end{array}\right] \Gamma_{2,2}\left[\begin{array}{c}
H \\
G
\end{array}\right] \tilde{\Gamma}_{4,4}\left[\begin{array}{c}
H \\
G
\end{array}\right]
\end{aligned}
$$

We have defined $\tilde{\Gamma}_{4,4}\left[\begin{array}{l}H \\ G\end{array}\right]$ as the contribution of the four twisted bosons. In the " $\mathcal{N}_{4}=4$ " sector, namely for $(H, G) \neq(0,0), \tilde{\Gamma}_{4,4}\left[\begin{array}{l}H \\ G\end{array}\right] \equiv \frac{1}{\eta^{4} \bar{\eta}^{4}} \Gamma_{4,4}\left[\begin{array}{c}H \\ G\end{array}\right]$, and (2.3) coincides with the partition function of the $\mathcal{N}_{4}=4$ freely-acting orbifold of [9]. In the untwisted, unprojected sector, $(H, G)=(0,0)$, it is the usual sum over lattice momenta. In the non-compact limit, it is given instead by:

$$
\tilde{\Gamma}_{4,4}\left[\begin{array}{l}
0 \\
0
\end{array}\right] \equiv \frac{1}{\operatorname{Im} \tau^{2} \eta^{4} \bar{\eta}^{4}}
$$

\footnotetext{
${ }^{2}$ See for instance Ref. [14] for a review.
} 
In the lattice contribution of the two-torus, $\Gamma_{2,2}\left[\begin{array}{l}H \\ G\end{array}\right]$, the arguments indicate the shift produced by the translation associated to the orbifold twist. The one loop contribution is computed by regularizing the infrared with the method of Ref. [17]. We obtain:

$$
\frac{16 \pi^{2}}{g_{\text {grav }}^{2}} \approx-\frac{2}{3} \log \operatorname{Im} U\left|\vartheta_{4}(U)\right|^{4}-b \log \mu / M,
$$

where $\approx$ indicates that in the above expression we discard terms suppressed at large $V_{(4)}$, The correction (2.5) has a logarithmic divergence in the limit $R_{6} \rightarrow \infty(\operatorname{Im} U \rightarrow \infty)$, where the $\mathcal{N}_{4}=8$ supersymmetry is restored. This logarithmic dependence can be removed with an appropriate choice of the infrared cut-offt.

\section{The $\mathcal{N}_{4}=2$ heterotic and type I duals}

The heterotic dual is constructed as a $T^{2} \times T^{4} / Z_{2}$ orbifold, in which the twist is accompanied by a $Z_{2}$ translation on $T^{2}$, producing a left-right symmetric shift of the momenta; modular invariance requires then an embedding of the spin connection into the gauge group. When the gauge group is broken to $U(1)^{16}$ by Wilson lines, the massless spectrum originating from the currents contains only sixteen vector multiplets and no hypermultiplets. At this point, it is easy to write the partition function and to compute the "regular" gravitational amplitude, obtained by correcting the " $R^{2}$ " term with appropriate gauge amplitude terms. An example of such a subtraction, valid for orbifolds with an equal number of vector and hypermultiplets originating from the $c=16$ currents, $N_{V}=N_{H}$, was discussed in Refs. [10, 13, 21].

The type I dual was first constructed in Ref. [2], as an orientifold of the type IIB string with an $\mathcal{N}_{4}=8$ supersymmetry spontaneously broken to $\mathcal{N}_{4}=4$. The freely acting projection $Z_{2}^{\mathrm{F}}$, that breaks supersymmetry on the type IIB "parent" string, acts as a leftright symmetric reflection on four coordinates of the target space and as a half-circumference translation in another circle. The translation is effectively introduced by a projection onto the quantum numbers of the lattice of momenta associated to that compact coordinate. Indeed, in Ref. [2] two such constructions were discussed. They are distinguished by the action of this projection on this lattice: either as $\frac{1+(-1)^{m}}{2}$ or as $\frac{1+(-1)^{n}}{2}$. One gets type I orientifolds with different properties: in the first case, the "momentum shift", one gets a model with gauge group $S O(16)$, entirely provided by open string states ending on D9-branes: no D5-branes are present. This model, called "Scherk-Schwarz breaking" in Ref. [2], is dual to the heterotic construction. In order to obtain hypermultiplets from the the D-branes massless spectrum, it is necessary to introduce a Wilson line, coupled to the supersymmetry breaking projection. In this case, the gauge group splits into factors $S O\left(N_{1}\right) \times S O\left(N_{2}\right)$, with hypermultiplets in the $\left(\mathbf{N}_{\mathbf{1}}, \mathbf{N}_{\mathbf{2}}\right)$, bifundamental representation. By introducing appropriate Wilson lines it is possible to break the gauge group to $U(1)^{16}$, and obtain the dual of the $\tilde{\Omega}$ orientifold presented in the previous section.

More subtle is the case of the "winding shift", otherwise called M-theory breaking in [2]. In this case, supersymmetry appears to be unbroken in the open string sector, that

\footnotetext{
${ }^{3}$ For more details about these issues, we refer the reader to [9, 13, 17, 18]- 20].
} 
provides the massless states of a $\mathcal{N}_{4}=4$ super Yang-Mills theory with maximal gauge group $S O(16) \times S O(16)$ : the first factor originates from D9-branes states, while the second factor originates from a D5-branes sector. It seems therefore that such construction cannot be dual to the other models we have considered. The problem is not constituted by the presence of supersymmetry in a part of the open string sector, because interactions with the other sectors communicate the breaking of supersymmetry also to this sector. However, even if at the end the massless spectra at the $U(1)$ most probably coincide, there is a mismatch between heterotic and type I constructions: in the latter, the D9- and D5-branes gauge groups have independent gauge couplings, while on the heterotic side, the full gauge group has the same coupling: $g^{-2} \approx\langle\operatorname{Im} S\rangle$, where $S$ is the heterotic dilaton-axion field. There is no loop renormalization, and non-perturbative corrections cannot produce a linear dependence such to dramatically correct the conclusions obtained by looking at the tree level contribution. We nevertheless keep on considering here also this construction, because our arguments, slightly modified, will enable us to obtain informations about the non-perturbative regime of this construction too.

\section{The $R^{2}$ corrections}

Both in the heterotic and in the type I models, the gravitational amplitude receives a contribution only at the tree and one loop levels. On the heterotic side, the one loop contribution is easily computed by inserting in the partition function appropriate operators; their action on the left-movers is that of the helicity operator, $Q^{2}$ (see Ref. [17]). After saturation of the fermion zero modes, their action on the right movers is reduced to a covariant differentiation on certain bloks of the " $\mathcal{N}_{4}=2$ " sector of the partition function, namely on the second helicity supertrace, $B_{2}$ f. This quantity possesses universality properties [22], and is fixed by the choice of the $Z_{2}$ shift on $T^{2}$ and by the value of the difference, $N_{V}-N_{H}$, of vector and hypermultiplets originating from the currents, in the untwisted sector of the orbifold. In our case $N_{V}-N_{H}=16$, and we have:

$$
B_{2}=\frac{1}{\bar{\eta}^{24}} \sum_{(H, G)}^{\prime} \Gamma_{2,2}\left[\begin{array}{l}
H \\
G
\end{array}\right] \bar{\Omega}\left[\begin{array}{l}
H \\
G
\end{array}\right]
$$

where the prime indicates that the sum is taken only over the values $(H, G)=\{(0,1),(1,0)$, $(1,1)\}$. The modular forms $\Omega\left[\begin{array}{l}H \\ G\end{array}\right]$ read:

$$
\begin{aligned}
& \Omega\left[\begin{array}{l}
0 \\
1
\end{array}\right]=\frac{1}{2}\left(\vartheta_{3}^{4}+\vartheta_{4}^{4}\right) \vartheta_{3}^{8} \vartheta_{4}^{8} \\
& \Omega\left[\begin{array}{l}
1 \\
0
\end{array}\right]=-\frac{1}{2}\left(\vartheta_{2}^{4}+\vartheta_{3}^{4}\right) \vartheta_{2}^{8} \vartheta_{3}^{8} \\
& \Omega\left[\begin{array}{l}
1 \\
1
\end{array}\right]=\frac{1}{2}\left(\vartheta_{2}^{4}-\vartheta_{4}^{4}\right) \vartheta_{2}^{8} \vartheta_{4}^{8} .
\end{aligned}
$$

\footnotetext{
${ }^{4}$ We refer the reader to Refs. [17, 19] for more details about these computations.
} 
The amplitude we need to compute is:

$$
\left\langle R^{2}\right\rangle_{(\mathrm{reg})}=\left\langle R^{2}\right\rangle+\frac{1}{12}\left\langle P^{2}\right\rangle_{\left(T^{2}\right)}+\frac{5}{48}\left\langle F^{2}\right\rangle_{\text {gauge }} .
$$

The first term on the r.h.s. is the ordinary $R^{2}$ amplitude, computed with the standard methods (see for instance [15, 17, 19]. The second and third term correspond respectively to the amplitude $F^{2}$ of the $U(1)^{2}$ of the two-torus, computed as in Ref. [13], and to the gauge amplitude $F^{2}$ of the currents; the latter is obtained by inserting in the one-loop vacuum amplitude the operator:

$$
P_{\text {(gauge) }}^{2}=Q^{2} \bar{P}_{\text {(gauge) }}^{2},
$$

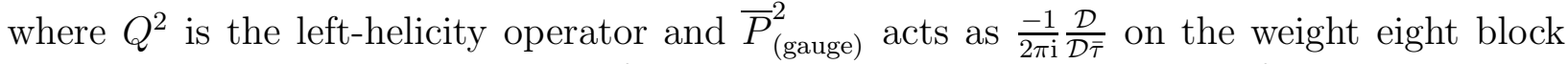
corresponding to the contribution of the $c=16$ lattice. The correction of the gravitational amplitude provided by the second and third term in the r.h.s. of (3.3) was shown in Ref. [13] to be necessary in order to subtract singularities that don't have a counterpart in dual string constructions, and to provide therefore a quantity suitable for a comparison of dual theories. In the present case, in the $(H, G) \neq(0,0)$ sector, this is given by:

$$
\frac{\bar{\vartheta}^{2}\left[\begin{array}{c}
1+H \\
1+G
\end{array}\right] \bar{\Omega}\left[\begin{array}{l}
H \\
G
\end{array}\right]}{4 \bar{\eta}^{6}}
$$

Acting on $B_{2}$ with the right-moving part of (3.3), $\bar{R}_{(\mathrm{reg})}^{2}$, we obtain:

$$
\bar{R}_{(\mathrm{reg})}^{2} B_{2}=\frac{2}{3} \sum_{(H, G)}^{\prime} \Gamma_{2,2}\left[\begin{array}{l}
H \\
G
\end{array}\right]
$$

The final result is given by the infrared-regularized integral of (3.6) on the fundamental domain. On the type I side, the gravitational corrections are proportional to an index [23], and the analog of (3.3) simply amounts to a proper choice of normalization. The analogous of (2.1) and (2.5) for the heterotic model reads therefore:

$$
\begin{aligned}
\frac{16 \pi^{2}}{g_{\text {grav }}^{2}(\mu)}= & 16 \pi^{2} \operatorname{Im} S-\frac{2}{3} \log \operatorname{Im} T\left|\vartheta_{4}(T)\right|^{4}-\frac{2}{3} \log \operatorname{Im} U\left|\vartheta_{4}(U)\right|^{4} \\
& -b \log \mu / M,
\end{aligned}
$$

where $S$ is the heterotic axion-dilaton field,

$$
\operatorname{Im} S=\frac{1}{g_{\text {Het }}^{2}}
$$

and the fields $T$ and $U$ are respectively the Kähler class and the complex structure moduli of the two-torus. For the parametrization of Eq. (2.2), the $\vartheta_{4}$ functions are obtained, as in Section 2, when the $Z_{2}$ translation in $T^{2}$ acts as a half-circumference translation, generated by the $(-1)^{m}$, along $x_{6}$. For the type I dual we have instead [10]:

$$
\frac{16 \pi^{2}}{g_{\text {grav }}^{2}(\mu)}=16 \pi^{2} \operatorname{Im} S-\frac{2}{3} \log \operatorname{Im} U\left|\vartheta_{4}(U)\right|^{4}-b \log \mu / M
$$


In both the expressions (3.7) and (3.9) we used the string scale $M \equiv 1 / \sqrt{\alpha^{\prime}}$ and the infrared cut-off $\mu$. These quantities can be either those of the heterotic or those of the type I string, their ratio being invariant under duality (cfr. Eq. (2.5)). On the type I side, $\operatorname{Im} S$ is associated to the coupling constant of the gauge fields originating from the D9-branes sector and $U$ is still the complex structure modulus of the untwisted two-torus. The heterotic volume modulus $T$, on the other hand, is dual to $\tilde{S}^{\prime} \equiv-1 / S^{\prime}$, where $S^{\prime}$ is the type I field whose VEV is the coupling constant of the gauge fields in the D5-branes sector [12]. There is therefore an apparent mismatch between expression (3.7) and (3.9). However, the functional dependence of (3.7) on this modulus is such that, for large $T$ (i.e. for small $S^{\prime}$ ), the dependence on this modulus disappears from the correction 0 . On the other hand, the opposite limit, $T \rightarrow 0$, with $U$ fixed, corresponds to a decompactification to a six dimensional "true orbifold limit", where the states with mass shifted by the translation associated to the orbifold twist become infinitely massive. In this limit we expect therefore to recover the properties of the orbifold discussed in [24]. In this limit, the correction (3.7) diverges linearly in $\operatorname{Im} \tilde{T} \sim \operatorname{Im} S^{\prime}$, in agreement with the appearance of a D5-branes sector. The limit in which the heterotic and type I models are comparable is therefore $T \rightarrow \infty$. The full $T$-dependence shown in (3.7) has then to be interpreted, on the type I side, as a nonperturbative correction. In the limit $T \rightarrow 0$, namely the orbifold limit, the properties of the theory change dramatically, and the spectrum has to be found by expanding around a new perturbative vacuum, namely that presented in Refs. [24].

We can now proceed to compare all the constructions through the corrections to the $R^{2}$ term. As we discussed, the two type I orientifolds, $\Omega$ and $\tilde{\Omega}$, are trivially dual the one to the other. The D9-branes sector of the $\Omega$ orientifold is therefore mapped under duality into the D5-branes sector of the $\tilde{\Omega}$ construction. In particular, the coupling of the D9-branes gauge fields is dual to the coupling of the D5-branes gauge fields. This coupling is parametrized by the vacuum expectation value of the imaginary part of a field, $S$, with:

$$
\operatorname{Im} S=\mathrm{e}^{-\phi_{4}} G^{1 / 4} \omega^{2} .
$$

In the dual construction, the coupling of the D5-branes gauge fields is parametrized by the v.e.v. of the imaginary part of a field $S^{\prime}$ (see also Refs. [12]), with:

$$
\operatorname{Im} S^{\prime}=\mathrm{e}^{-\phi_{4}} G^{1 / 4} \omega^{-2} .
$$

In (3.10) and (3.11), $\phi_{4}$ is the type I dilaton of four-dimensions, $\sqrt{G}$ the volume of $T^{2}$ and $\omega^{4}$ the volume of the K3 $\left(\sim T^{4} / Z_{2}\right)$ of the type I compactification. Under duality, $S \leftrightarrow S^{\prime}$, as it can be seen also by the comparison of (2.1) and (3.9). From (2.5) we can read then the asymptotic behavior of the $\Omega$ orientifold in the small- $S$ limit. We learn that the contribution of this field vanishes in this limit, signaling the breaking of S-duality. By further comparison with the $R^{2}$ correction of the heterotic dual, given in (3.7), we can make an ansatz for the complete correction to the effective coupling of this term. By considering the "vanishing" of the contribution of any of the fields $S, \tilde{T} \equiv-1 / T, \tilde{U} \equiv-1 / U$ in the small fields limits, as opposed to the linear divergence when these fields are large, we argue that the full nonperturbative extension of the gravitational correction (3.7) is given by the symmetrization

\footnotetext{
${ }^{5}$ More precisely, the string threshold diverges only logarithmically in $T$.
} 
of that expression in the fields $\tilde{S}, T, U$ :

$$
\begin{aligned}
\frac{16 \pi^{2}}{g_{\text {grav }}^{2}} \approx & -\frac{2}{3} \log \operatorname{Im} \tilde{S}\left|\vartheta_{4}(\tilde{S})\right|^{4}-\frac{2}{3} \log \operatorname{Im} T\left|\vartheta_{4}(T)\right|^{4}-\frac{2}{3} \log \operatorname{Im} U\left|\vartheta_{4}(U)\right|^{4} \\
& +\mathcal{E}(\tilde{S}, T, U)
\end{aligned}
$$

where $\mathcal{E}(\tilde{S}, T, U)$ stays for a series of exponentials of the type $\mathrm{e}^{\mathrm{i}\left(k_{1} \tilde{S}+k_{2} T+k_{3} U\right)}$, in which the fields $\tilde{S}, T, U$ appear weighted by "instanton numbers". This term, symmetric in $\tilde{S}, T, U$, is suppressed in both the large and small fields limit]. As a consequence, also the dependence on the field $S$, linearly divergent for large $\operatorname{Im} S$, is instead only logarithmic when $S$ is small 8 . Note that if in Eq. (3.12) we take the limit $\tilde{S} \rightarrow \infty$ (i.e. $S \rightarrow 0$ ), we obtain:

$$
\frac{16 \pi^{2}}{g_{\text {grav }}^{2}} \approx-\frac{2}{3} \log \operatorname{Im} T\left|\vartheta_{4}(T)\right|^{4}-\frac{2}{3} \log \operatorname{Im} U\left|\vartheta_{4}(U)\right|^{4}+\mathcal{O}(\log \operatorname{Im} S)
$$

In this limit, the effective coupling indeed looks like the non-perturbative expression of the coupling of an $\mathcal{N}_{4}=2$ theory with only D5-branes. We can in fact interpret, as usual, the logarithmic dependence on the field, $S$, as the remnant of non-perturbative effects, that corrects the absence of any perturbative term.

The limit $\tilde{T} \equiv-1 / T \rightarrow \infty, \tilde{T}<S$, is a perturbative limit on the heterotic side. However, as we argued, it should correspond to a limit of the theory in which D5-branes massless states are expected to appear. As long as $\operatorname{Im} \tilde{T} \ll \operatorname{Im} S$, these states are essentially decoupled from the theory. As we approach the border of the heterotic perturbative regime, these states start to be dynamical, and they cannot be neglected. Heterotic/type I duality in that regime must be studied in the framework of the construction of Refs. [24]: we argue that the appearance of a non-negligible $T$-dependence on the threshold corrections accounts for the "transition" to this phase of the theory. In the opposite limit, of $\mathcal{N}_{4}=4$ supersymmetry restoration, this sector instead decouples.

For the "winding breaking" model, owing to the presence of both D9- and D5-branes sectors, the analogous of (3.9) reads:

$$
\frac{16 \pi^{2}}{g_{\text {grav }}^{2}(\mu)}=16 \pi^{2} \operatorname{Im} S+16 \pi^{2} \operatorname{Im} S^{\prime}-\frac{2}{3} \log \operatorname{Im} U\left|\vartheta_{4}(U)\right|^{4}-b \log \mu / M .
$$

In this case, we cannot derive the strong coupling behavior of the threshold correction by looking at an heterotic dual. A naive investigation of the decompactification limit in the $\tilde{\Omega}$ dual orientifold doesn't help either, because, owing to the simultaneous presence of both D9- and D5-branes, this is in any case a singular limit. However, also in this model the

\footnotetext{
${ }^{6}$ To be more precise, we should introduce here the fields $\tau_{S} \equiv 4 \pi S$ and $\tilde{\tau}_{S} \equiv \tau_{S}^{-1}$, and normalize the argument in the first term of the correction according to: $\vartheta_{4}\left(6 \tilde{\tau}_{S}\right)$. However, for the sake of simplicity, in this and in the subsequent formulae we don't care about the normalization of the fields.

${ }^{7}$ The limit in which these fields are small must in fact be taken after a Poisson resummation, that converts this sum of exponentials into another sum of exponentials of the inverse fields, still suppressed.

${ }^{8}$ We recall that under $-1 / S \rightarrow S, \vartheta_{4}(-1 / S) \rightarrow \vartheta_{2}(S)$.
} 
$\mathcal{N}_{4}=2$ supersymmetry comes from the spontaneous breaking of $\mathcal{N}_{4}=4$, which is restored in a corner of the moduli space. In this phase, the D5-branes sector of the $\Omega$ orientifold is expected to disappear, and the properties of the theory should be analogous to those of the $\mathcal{N}_{4}=4$ phase of the "momentum breaking" model. We argue therefore that, as in that case, also here the $S \rightarrow-1 / S$ and $S^{\prime} \rightarrow-1 / S^{\prime}$ symmetries are broken, and the approximate behavior of the $R^{2}$ threshold correction is as in (3.12).

\section{Reduced rank type I models and duality}

An analogous non-perturbative behavior is shown by other string constructions, with a gauge group of reduced rank, $r=8$ and $r=4$. Of these theories, we know only the type I, $\Omega$ orientifold constructions, and their $\tilde{\Omega}$ duals. On the type I side, the reduction of the rank is obtained by introducing a non-vanishing quantized antisymmetric NS-NS tensor $B_{a b}$. Examples with $\mathcal{N}_{4}=4$ supersymmetry have been constructed in Ref. [3, \#. This construction has then been extended to $\mathcal{N}_{4}=2, Z_{2}$ non freely-acting orbifolds [5, [6]. Here we are interested in the case in which the $Z_{2}$ projection that partially breaks supersymmetry acts freely, as in Ref. [2]. In this way, with a rank 2 antisymmetric tensor we obtain a "Scherk-Schwarz breaking" model with maximal gauge group $S O(16)$ and an "M-theory breaking" model with maximal gauge group $S O(8)_{(9)} \times S O(8)_{(5)}$. With an antisymmetric tensor of rank four we obtain then a "Scherk-Schwarz breaking" model with maximal gauge group $S O(8)$ and an "M-theory breaking" model with maximal gauge group $S O(4)_{(9)} \times$ $S O(4)_{(5)}$. With appropriate Wilson lines, the above $S O(N)$ groups can be reduced to the corresponding $U(N / 2)$. As for the rank 16 of the previous section, Wilson lines are needed in order to introduce hypermultiplets in the "Scherk-Schwarz breaking" models, besides the four originating from the compact space.

The Klein Bottle, Annulus and Möbius strip amplitudes for these models can be easily derived from the corresponding amplitudes of the "Scherk-Schwarz" - and "M-theory"-breaking $\mathcal{N}_{4}=2$ models of Ref.[2]. In our case, we work in four dimensions, and the shifted lattice sum terms $Z_{m}$, defined in Ref. [25], have to be adapted for a two-dimensional lattice. The models under consideration can be constructed also in five dimension, because only one direction in the two-torus is shifted: we can therefore always factorize the sum $\Gamma_{(1)}$ over the unshifted momenta of the lattice of one of the two circles. In the following, by $Z_{m}$ we will actually mean $\Gamma_{(1)} \times Z_{m}$. For the "Scherk-Schwarz" breaking, we have in this case ?:

$$
\mathcal{K}=\sum_{m} \frac{1}{4}\left(Q_{O}+Q_{V}\right)\left(q^{2}\right)\left[P Z_{m}+\tilde{W}(-1)^{m} Z_{m}\right]
$$

where $q=\mathrm{e}^{-2 \pi t}, P$ is the momentum sum of the lattice of $T^{4}$ :

$$
P=\sum_{m} \frac{q^{\frac{\alpha^{\prime}}{2} m^{T} g^{-1} m}}{\eta^{4}\left(q^{2}\right)}
$$

\footnotetext{
${ }^{9}$ For a definition of the characters $Q_{0}, Q_{V}$ we refer to [2].
} 
and $\tilde{W}$ is the corresponding winding sum, in which the windings satisfy the constraint imposed by a quantized non-vanishing antisymmetric tensor [3, 田, 娄]:

$$
\frac{2}{\alpha^{\prime}} B_{a b} n^{b}=2 m_{a}
$$

so that

$$
\tilde{W}=2^{-4} \sum_{\epsilon=0,1} \sum_{n} \frac{q^{\frac{1}{2 \alpha^{\prime}} n^{T} g n} \mathrm{e}^{\frac{2 i \pi}{\alpha^{\prime}} n^{T} B \epsilon}}{\eta^{4}\left(q^{2}\right)},
$$

where the sum over $\epsilon$, a set of vectors with entries 0 or 1, introduces the projection along the directions of non-vanishing $B_{a b}$. The Annulus amplitude reads:

$$
\begin{aligned}
\mathcal{A}=\sum_{m} & \left\{\frac{\left(N_{1}+N_{2}\right)^{2}}{4}\left(Q_{O}+Q_{V}\right)(\sqrt{q}) 2^{r-4} \tilde{P} Z_{m}\right. \\
& \left.-\frac{\left(N_{1}-N_{2}\right)^{2}}{4}\left(Q_{O}-Q_{V}\right)(\sqrt{q})\left(\frac{\vartheta_{3}^{2} \vartheta_{4}^{2}}{\eta^{4}}\right)(\sqrt{q})(-1)^{m} Z_{m}\right\},
\end{aligned}
$$

where

$$
\tilde{P} \equiv \sum_{\epsilon=0,1} \sum_{m} \frac{q^{\frac{\alpha^{\prime}}{2}\left(m+\frac{1}{\alpha^{\prime}} B \epsilon\right)^{T} g^{-1}\left(m+\frac{1}{\alpha^{\prime}} B \epsilon\right)}}{\eta^{4}(\sqrt{q})}
$$

and $r$ is the rank of $B_{a b}$. Finally, the Möbius strip amplitude is given by:

$$
\begin{array}{r}
\mathcal{M}=-\frac{\left(N_{1}+N_{2}\right)}{4} \sum_{m}\left\{\left(\hat{Q}_{O}+\hat{Q}_{V}\right)(-\sqrt{q}) 2^{(r-4) / 2} \hat{\tilde{P}} Z_{m}+\right. \\
\left.-\left(\hat{Q}_{O}-\hat{Q}_{V}\right)(-\sqrt{q})\left(\frac{\hat{\vartheta}_{3}^{2} \hat{\vartheta}_{4}^{2}}{\hat{\eta}^{4}}\right)(-\sqrt{q})(-1)^{m} Z_{m}\right\},
\end{array}
$$

with

$$
\hat{\tilde{P}} \equiv \sum_{\epsilon=0,1} \sum_{m} \frac{q^{\frac{\alpha^{\prime}}{2}\left(m+\frac{1}{\alpha^{\prime}} B \epsilon\right)^{T} g^{-1}\left(m+\frac{1}{\alpha^{\prime}} B \epsilon\right)} \gamma_{\epsilon}}{\hat{\eta}^{4}(-\sqrt{q})} .
$$

$\gamma_{\epsilon}$ is a cocycle, necessary in order to ensure a correct particle interpretation (see [6]). Notice that the projection introduced by $B_{a b}$ acts on the momenta of $T^{4}$, while the translation due to the supersymmetry breaking $Z_{2}^{\mathrm{F}}$ projection acts on the momenta of the extra $T^{2}$. The ChanPaton factors are then fixed by the vanishing condition for the infrared divergences appearing in the so-called "transverse channel", in which the open string diagrams are interpreted as diagrams for the propagation of a closed string. The proper "time" $\ell$ in the transverse channel is related to the time $t$ in the direct channel in a different way for the three topologies, Klein bottle, Annulus and Möbius strip:

$$
\begin{aligned}
\mathcal{K} & : \quad \ell=\frac{1}{2 t_{\mathcal{K}}}, \\
\mathcal{A} & : \quad \ell=\frac{2}{t_{\mathcal{A}}}, \\
\mathcal{M} & : \quad \ell=\frac{1}{2 t_{\mathcal{M}}} .
\end{aligned}
$$


By transforming therefore the above amplitudes to the transverse channel and looking at the divergence at the origin of the lattice $(\ell \rightarrow \infty)$, it is easy to see that the tadpole condition is modified: with respect to the freely acting orbifold without antisymmetric tensor, now the rank of the gauge group is reduced by a factor $r / 2$. In the transverse channel, after taking the $\ell \rightarrow \infty$ limit, we obtain the following contributions to the infrared divergence:

$$
\begin{aligned}
\tilde{\mathcal{K}}_{0} & =\frac{2^{5}}{4 \times 2} V_{(6)} \\
\tilde{\mathcal{A}}_{0} & =\frac{2^{(r-4)-5}}{4 \times 2}\left(N_{1}+N_{2}\right)^{2} V_{(6)} \\
\tilde{\mathcal{M}}_{0} & =-\frac{2 \times 2^{(r-4) / 2}}{4 \times 2}\left(N_{1}+N_{2}\right) V_{(6)}
\end{aligned}
$$

where

$$
V_{(6)}=\sqrt{\operatorname{det}\left(g_{(6)} / \alpha^{\prime}\right)}
$$

They are canceled if:

$$
\left[2^{r / 2}\left(N_{1}+N_{2}\right)-2^{5}\right]^{2}=0
$$

that means

$$
\left(N_{1}+N_{2}\right)=\frac{2^{5}}{2^{r / 2}}
$$

In the above amplitudes, the splitting of the Chan-Paton factors into $N_{1}$ and $N_{2}$ is due to a Wilson line with entries $\frac{1}{2}$, that can be chosen such that $N_{1}=N_{2}=N=32 / 2^{r / 2}$. In this case, the vector multiplets are in the $(\mathbf{N}(\mathbf{N}-\mathbf{1}) / \mathbf{2}, \mathbf{1}) \oplus(\mathbf{1}, \mathbf{N}(\mathbf{N}-\mathbf{1}) / \mathbf{2})$ adjoint representations of $S O(N) \times S O(N)$, the hypermultiplets in the bifundamental $(\mathbf{N}, \mathbf{N})$. The case of our interest is obtained when a further Wilson line is introduced, with entries $\frac{1}{4}$, that breaks the gauge group to its unitary subgroup, $U(N / 2) \times U(N / 2)$.

In the case of "M-theory" breaking, the amplitudes are, in the simplest case without Wilson lines:

$$
\begin{gathered}
\mathcal{K}=\sum_{m}\left\{\frac{1}{4}\left(Q_{O}+Q_{V}\right)\left(q^{2}\right)(P+\tilde{W}) Z_{m}+\frac{2^{(4-r) / 2}}{2}\left(Q_{S}+Q_{C}\right)\left(q^{2}\right)\left(\frac{\vartheta_{2}^{2} \vartheta_{3}^{2}}{\eta^{4}}\right)\left(q^{2}\right) Z_{m+\frac{1}{2}}\right\} \\
\mathcal{A}=\frac{1}{4} \sum_{m}\left\{\left[\left(Q_{O}+Q_{V}\right)(\sqrt{q})\left(2^{r-4} n^{2} \tilde{P}+d^{2} W\right)\right] Z_{2 m}\right. \\
\left.+\frac{2^{r / 2}}{2}(n d)\left(Q_{S}+Q_{C}\right)(\sqrt{q})\left(\frac{\vartheta_{2}^{2} \vartheta_{3}^{2}}{\eta^{4}}\right)(\sqrt{q})\right\} Z_{2 m+1} \\
\mathcal{M}=-\frac{1}{4} \sum_{m}\left\{\left(\hat{Q}_{O}+\hat{Q}_{V}\right)(-\sqrt{q})\left[2^{(r-4) / 2} n \hat{\tilde{P}}+2^{-2} d \hat{\tilde{W}}\right] Z_{4 m}\right. \\
\left.-(n+d)\left(\hat{Q}_{O}-\hat{Q}_{V}\right)(-\sqrt{q})\left(\frac{\hat{\vartheta}_{3}^{2} \hat{\vartheta}_{4}^{2}}{\hat{\eta}^{4}}\right)(-\sqrt{q}) Z_{4 m+2}\right\}
\end{gathered}
$$


where

$$
\hat{\tilde{W}}=\sum_{\epsilon=0,1} \sum_{n} \frac{q^{\frac{1}{2 \alpha^{\prime}} n^{\mathrm{T}} g n} \mathrm{e}^{\frac{2 i \pi}{\alpha^{\prime}} n^{\mathrm{T}} B \epsilon} \tilde{\gamma}_{\epsilon}}{\hat{\eta}^{4}(-\sqrt{q})},
$$

with $\tilde{\gamma}_{\epsilon}$ a further, independent cocycle (see Ref. [6]). In this case, the further reduction of the rank of the gauge group involves the parts coming both from the D9-branes and the D5-branes sectors, in a symmetric way. The contributions to the infrared divergence are:

$$
\begin{aligned}
\tilde{\mathcal{K}}_{0} & =\frac{2^{5}}{4 \times 2} V_{(2)}\left[V_{(4)}+\frac{2^{-r}}{V_{(4)}}\right] \\
\tilde{\mathcal{A}}_{0} & =\frac{2^{-5}}{4 \times 2} V_{(2)}\left[V_{(4)} 2^{r} n^{2}+\frac{d^{2}}{V_{(4)}}\right] \\
\tilde{\mathcal{M}}_{0} & =-\frac{2}{4 \times 2} V_{(2)}\left[V_{(4)} 2^{r / 2} n+\frac{2^{-r / 2} d^{2}}{V_{(4)}}\right],
\end{aligned}
$$

where $V_{(2)}=\sqrt{\operatorname{det}\left(g_{(2)} / \alpha^{\prime}\right)}$ and $V_{(4)}=\sqrt{\operatorname{det}\left(g_{(4)} / \alpha^{\prime}\right)}$ are the volumes of the two- and the four-torus respectively. The tadpole cancellation conditions are:

$$
n=\frac{2^{5}}{2^{r / 2}}, \quad d=\frac{2^{5}}{2^{r / 2}}
$$

The construction of the $\tilde{\Omega}$ orientifolds is analogous, and their partition functions are obtained by those of the ordinary orientifolds, by exchanging the role of D9- and D5-branes. The description from the bulk viewpoint, suitable to investigate the decompactification limit, is then obtained by shifting all the windings and doubling all the momenta of the coordinates transverse to the D5-branes in the Klein bottle and in the open string diagrams. As before, Wilson lines are needed in order to break the gauge group to the Abelian subgroup, and obtain duality between type I gauge groups and the $\tilde{\Omega}$ constructions with the D5-branes sitting on the corresponding $\mathrm{O} 5$ orientifold plane, in order to have a local cancellation of the tadpoles. In this case, however, we don't have the heterotic dual orbifold constructions: the only heterotic orbifolds with reduced rank of the gauge group in which the gravitational corrections have a perturbative expression like (3.7), with a factorization of the moduli $T$ and $U$, are those considered in Refs. 13 四: they are necessarily constructed at a point in the moduli space in which the higher level (= reduced rank) gauge group is realized with real fermions, the points $\left[S U(2)_{\kappa}\right]^{16 / \kappa}=\left[S O(3)_{\kappa / 2}\right]^{16 / \kappa}$. In these heterotic orbifolds, the massless states originating from the currents have an effective $\mathcal{N}_{4}=4$ supersymmetry. They are dual to type IIA orbifolds with spontaneously broken $\mathcal{N}_{4}=4$ supersymmetry, that are singular limits in the moduli space of K3 fibrations. The full, non-perturbative gravitational correction can be computed perturbatively on the type IIA side (see Refs. [13]), and it turns out that there is no limit in the space of moduli $S, T, U$ in which there is a restoration of the $\mathcal{N}_{4}=8$ supersymmetry.

\footnotetext{
${ }^{10}$ Heterotic constructions with gauge groups of reduced rank have also been considered in Ref. [26], and their relation to type I constructions in Refs. [3, 4,27 .
} 
Even without the heterotic duals, from which we would learn something more about one of the moduli, the comparison of " $\Omega$ " and " $\Omega$ " constructions allows us to see the spontaneous breaking of the $\mathcal{N}_{4}=8$ supersymmetry, as it was for the previous case with gauge group of rank 16. By looking at the various limits of these theories, with and without either D9- or D5-branes, we conclude that the gravitational corrections have an expression analogous to (3.12).

A note on the effective gauge coupling

The rank reduction indeed corresponds to a raising of the level of the algebra of the gauge group. In order to define the level, we don't need in fact to work with an explicit realization via Kač-Moody characters: the level can be intrinsically defined also through the strength of the coupling of the gauge group. Since the rank is halved when two isomorphic factors of the gauge group are identified (this is the effect of the operation that leaves massless the states invariant under the exchange of these factors and lifts the mass of the states which are odd), the level is doubled. The relation between level $\kappa$ and level 1 coupling is:

$$
\frac{1}{g_{\kappa}^{2}}=\frac{\kappa}{g_{(\kappa=1)}^{2}} \propto \frac{1}{g_{(\kappa=1)}^{2} N},
$$

where $N$ is the "effective" number of D9- or D5-branes, namely the number of branes modulo $Z_{2}$, level-raising identifications. Raising the level corresponds therefore to weakening the effective gauge coupling $g_{Y M} \equiv g_{\kappa} \propto g_{(\kappa=1)} \sqrt{N}$. If we now extrapolate from Eq. (4.19) and treat $\kappa$ and $N$ as parameters that can take any integer value, we can consider arbitrary high levels. This leads to a decoupling of the Yang Mills fields, that tend to be frozen to constant values: this is the no branes limit, with no gauge group. In the opposite limit, $N \rightarrow \infty, g_{Y M}$ is very big, and the Yang Mills fields dominate over the other fields: this is the limit of decoupling of gravity, whose coupling is $g \equiv g_{(\kappa=1)}$. We can look at this limit in another, equivalent way: we can rescale the couplings is such a way that the effective gauge coupling remains fixed. In the large $N$ limit we have therefore $g \rightarrow 0$, with $g^{2} N$ fixed. This is precisely the 't Hooft limit; in the literature, what we call the effective level $-\kappa$ gauge coupling is indicated by $\Lambda_{Q C D}$. In the theories we are considering, there are D9and D5-branes, with different four-dimensional coupling. The above decoupling argument applies separately for both the D9- and D5-branes sectors. When both the types of branes are present, the effective coupling of the bulk fields is in fact a combination of the two:

$g_{\text {bulk }}^{-2} \sim g_{(9)}^{-2}+g_{(5)}^{-2}$, and the effective couplings of the two parts of the gauge group are $g_{Y M(9)} \propto g_{(9)} \sqrt{N}, g_{Y M(5)} \propto g_{(5)} \sqrt{N}$.

\section{Conclusions}

In this work we have investigated the non-perturbative behavior of a class of four dimensional string models with $\mathcal{N}_{4}=2$ supersymmetry, in which there is a spontaneous breaking of $\mathcal{N}_{4}=8$. Some of these theories have dual realizations as freely acting orbifolds of both the heterotic and the type I string, others have only a type I orbifold realization. The 
spontaneous breaking of the $\mathcal{N}_{4}=8$ supersymmetry is due to a super-Higgs mechanism, nonperturbative in all the dual constructions: the supersymmetry restoration can be observed by comparing the heterotic and type I effective theories with the duals constructed as freely acting orbifolds of the type IIB string orientifolded with $\tilde{\Omega}$, the product of the world-sheet parity times the target-space reflection along four coordinates, compactified on $T^{4}$. In these constructions, the role of D9- and D5-branes is exchanged, as compared to the ordinary type I orientifolds. Owing to the free action of the orbifold projection, these theories possess phases in which only D5-branes appear. In these configurations, it is possible to investigate the limit in which the space transverse to the D5-branes is decompactified. Owing to the breaking of T-duality associated to the supersymmetry breaking projection produced by the D5-branes, this is a limit in which, from the bulk point of view, namely at large distance from the D-branes, there is an approximate restoration of the maximal amount of supersymmetry.

We have compared the dual constructions by looking at the renormalization of the effective coupling of the $R^{2}$ term, that depends on the moduli of the vector manifold, and therefore on the heterotic dilaton-axion field and on the type I vector coupling fields, " $S$ " and " $S^{\prime \prime}$ ". Since the $\mathcal{N}_{4}=4$ heterotic string possesses S-duality [28, the existence of a

spontaneous breaking of $\mathcal{N}_{4}=8$, that can be restored at the strong coupling, means that, in this case, the projection that breaks the $\mathcal{N}_{4}=4$ supersymmetry to $\mathcal{N}_{4}=2$ has an action also on $S$, the heterotic dilaton-axion field.

For simplicity, we have limited the analysis of these theories to the region of the moduli space in which the gauge group is broken to its Abelian subgroup. However, the "decompactification" arguments that led us to conclusions about their non-perturbative behavior can be applied also in regions in which the gauge group is non-Abelian. The key point is that the gauge group must correspond to a choice of Wilson lines dualizable to a Dp-branes configuration, $\mathrm{p}<9$, in which there is a local cancellation of the orientifold charge, without dilaton gradient along some transverse directions.

\section{Acknowledgements}

I thank C. Angelantonj, C. Bachas, K. Benakli, C. Kounnas, Y. Oz, P. Mayr and A. Zaffaroni for valuable discussions, the École Normale of Paris for kind hospitality, the École Polytechnique and the CNRS for kind hospitality and financial support. I want to thank also J.P. Derendinger for interesting discussions and hospitality in the University of Neuchâtel, where part of this work was carried out. This work was partially supported also by the Swiss National Science Foundation and the Swiss Office for Education and Science. 


\section{Appendix: The transverse channels for the reduced rank type I models}

We quote here the Klein Bottle, Annulus and Möbius Strip contributions in the transverse

channel for the reduced rank type I models of Section 4. For the rank reductions of the Scherk-Schwarz model of Ref. [2], we have:

$$
\begin{aligned}
& \tilde{\mathcal{K}}=\frac{2^{5}}{4}\left(Q_{O}+Q_{V}\right)(\mathrm{i} \ell)\left[V_{(4)} \sum_{n} \frac{\left(\mathrm{e}^{-2 \pi \ell}\right)^{\frac{1}{\alpha^{\prime}} n^{\mathrm{T}} g n}}{\eta^{4}(\mathrm{i} \ell)} V_{(2)}\left(\sum_{n^{\prime}} \tilde{Z}_{2 n^{\prime}}\right)+\right. \\
& \left.+\frac{2^{-4}}{V_{(4)}} \sum_{\epsilon=0,1} \sum_{m} \frac{\left(\mathrm{e}^{-2 \pi \ell}\right)^{\alpha^{\prime}\left(m+\frac{1}{\alpha^{\prime}} B \epsilon\right)^{\mathrm{T}} g^{-1}\left(m+\frac{1}{\alpha^{\prime}} B \epsilon\right)}}{\eta^{4}(\mathrm{i} \ell)} V_{(2)}\left(\sum_{n^{\prime}} \tilde{Z}_{2 n^{\prime}+1}\right)\right] \text {, } \\
& \tilde{\mathcal{A}}=\frac{2^{-5}}{4}\left\{\left(Q_{O}+Q_{V}\right)(\mathrm{i} \ell) 2^{r-4} V_{(4)}\left(N_{1}^{2}+N_{2}^{2}\right) \sum_{\epsilon=0,1} \sum_{n} \frac{\left(\mathrm{e}^{-2 \pi \ell}\right)^{\frac{1}{4 \alpha^{\prime}} n^{\mathrm{T}} g n} \mathrm{e}^{\frac{2 \mathrm{i} \pi}{\alpha^{\prime}} n^{\mathrm{T}} B \epsilon}}{\eta^{4}(\mathrm{i} \ell)} \times\right. \\
& \times V_{(2)}\left(\sum_{n^{\prime}} \tilde{Z}_{2 n^{\prime}}\right)+ \\
& \left.+4\left(N_{1}^{2}-N_{2}^{2}\right)\left(Q_{S}+Q_{C}\right)(\mathrm{i} \ell)\left(\frac{\vartheta_{2}^{2} \vartheta_{3}^{2}}{\eta^{4}}\right)(\mathrm{i} \ell) V_{(2)}\left(\sum_{n^{\prime}} \tilde{Z}_{2 n^{\prime}+1}\right)\right\}, \\
& \tilde{\mathcal{M}}=-\frac{2}{4}\left(N_{1}+N_{2}\right)\left\{\left(\hat{Q}_{O}+\hat{Q}_{V}\right)\left(\mathrm{i} \ell+\frac{1}{2}\right) 2^{(r-4) / 2} V_{(4)} \times\right. \\
& \times \sum_{\epsilon=0,1} \sum_{n} \frac{\left(\mathrm{e}^{-2 \pi \ell}\right)^{\frac{1}{\alpha^{\prime}} n^{\mathrm{T}} g n} \mathrm{e}^{\frac{2 \mathrm{i} \pi}{\alpha^{\prime}} n^{\mathrm{T}} B \epsilon} \gamma_{\epsilon}}{\hat{\eta}^{4}\left(\mathrm{i} \ell+\frac{1}{2}\right)} V_{(2)}\left(\sum_{n^{\prime}} \tilde{Z}_{2 n^{\prime}}\right)+ \\
& \left.+\left(\hat{Q}_{O}-\hat{Q}_{V}\right)\left(\mathrm{i} \ell+\frac{1}{2}\right)\left(\frac{\hat{\vartheta}_{3}^{2} \hat{\vartheta}_{4}^{2}}{\hat{\eta}^{4}}\right)\left(\mathrm{i} \ell+\frac{1}{2}\right) V_{(2)}\left(\sum_{n^{\prime}} \tilde{Z}_{2 n^{\prime}+1}\right)\right\} .
\end{aligned}
$$

For the "M-theory breaking" model we have:

$$
\begin{aligned}
\tilde{\mathcal{K}}= & \frac{2^{5}}{4}\left(Q_{O}+Q_{V}\right)(\mathrm{i} \ell)\left[V_{(4)} \sum_{n} \frac{\left(\mathrm{e}^{-2 \pi \ell}\right)^{\frac{1}{\alpha^{\prime}} n^{\mathrm{T}} g n}}{\eta^{4}(\mathrm{i} \ell)}+\right. \\
& \left.+\frac{2^{-4}}{V_{(4)}} \sum_{\epsilon=0,1} \sum_{m} \frac{\left(\mathrm{e}^{-2 \pi \ell}\right)^{\alpha^{\prime}\left(m+\frac{1}{\alpha^{\prime}} B \epsilon\right)^{\mathrm{T}} g^{-1}\left(m+\frac{1}{\alpha^{\prime}} B \epsilon\right)}}{\eta^{4}(\mathrm{i} \ell)}\right] V_{(2)}\left(\sum_{n^{\prime}} \tilde{Z}_{2 n^{\prime}}\right)+ \\
+ & \frac{2^{5-r / 2}}{2}\left(Q_{O}-Q_{V}\right)(\mathrm{i} \ell)\left(\frac{\vartheta_{3}^{2} \vartheta_{4}^{2}}{\eta^{4}}\right)(\mathrm{i} \ell) V_{(2)}\left(\sum_{n^{\prime}}(-1)^{n^{\prime}} \tilde{Z}_{2 n^{\prime}}\right)
\end{aligned}
$$




$$
\begin{aligned}
\tilde{\mathcal{A}}= & \frac{2^{-5}}{4}\left\{( Q _ { O } + Q _ { V } ) ( \mathrm { i } \ell ) \left[2^{r-4} V_{(4)} n^{2} \sum_{\epsilon=0,1} \sum_{n} \frac{\left(\mathrm{e}^{-2 \pi \ell}\right)^{\frac{1}{4 \alpha^{\prime}} n^{\mathrm{T}} g n} \mathrm{e}^{\frac{2 \mathrm{i} \pi}{\alpha^{\prime}} n^{\mathrm{T}} B \epsilon}}{\eta^{4}(\mathrm{i} \ell)}+\right.\right. \\
& \left.+\frac{1}{V_{(4)}} d^{2} \frac{\left(\mathrm{e}^{-2 \pi \ell}\right)^{\frac{\alpha^{\prime}}{4} m^{\mathrm{T}} g^{-1} m}}{\eta^{4}(\mathrm{i} \ell)}\right] V_{(2)}\left(\sum_{n^{\prime}} \tilde{Z}_{2 n^{\prime}}\right)+ \\
& \left.+2 \times 2^{r / 2}(n d)\left(Q_{O}-Q_{V}\right)(\mathrm{i} \ell)\left(\frac{\vartheta_{3}^{2} \vartheta_{4}^{2}}{\eta^{4}}\right)(\mathrm{i} \ell) V_{(2)}\left(\sum_{n^{\prime}}(-1)^{n^{\prime}} \tilde{Z}_{2 n^{\prime}}\right)\right\}, \\
\tilde{\mathcal{M}}=- & \frac{2}{4}\left\{( \hat { Q } _ { O } + \hat { Q } _ { V } ) ( \mathrm { i } \ell + \frac { 1 } { 2 } ) \left[2^{(r-4) / 2} V_{(4)} n \sum_{\epsilon=0,1} \sum_{n} \frac{\left(\mathrm{e}^{-2 \pi \ell}\right)^{\frac{1}{\alpha^{\prime}} n^{\mathrm{T}} g n} \mathrm{e}^{\frac{2 \mathrm{i} \pi}{\alpha^{\prime}} n^{\mathrm{T}} B \epsilon} \hat{\eta}^{4}\left(\mathrm{i} \ell+\frac{1}{2}\right)}{+}+\right.\right. \\
& +\frac{2^{-2}}{V_{(4)}} d \sum_{\epsilon=0,1} \sum_{m} \frac{\left(\mathrm{e}^{-2 \pi \ell}\right)^{\alpha^{\prime}\left(m+\frac{1}{\alpha^{\prime}} B \epsilon\right)^{\mathrm{T}} g^{-1}\left(m+\frac{1}{\alpha^{\prime}} B \epsilon\right)} \tilde{\gamma}_{\epsilon}}{\hat{\eta}^{4}\left(\mathrm{i} \ell+\frac{1}{2}\right)} V_{(2)}\left(\sum_{n^{\prime}} \tilde{Z}_{2 n^{\prime}}\right)+ \\
+ & \left.\left(\hat{Q}_{O}-\hat{Q}_{V}\right)\left(\mathrm{i} \ell+\frac{1}{2}\right)\left(\frac{\hat{\vartheta}_{3}^{2} \hat{\vartheta}_{4}^{2}}{\hat{\eta}^{4}}\right)\left(\mathrm{i} \ell+\frac{1}{2}\right)(n+d) V_{(2)}\left(\sum_{n^{\prime}}(-1)^{n^{\prime}} \tilde{Z}_{2 n^{\prime}}\right)\right\},
\end{aligned}
$$

where

$$
V_{(4)}=\sqrt{\operatorname{det}\left(g_{(4)} / \alpha^{\prime}\right)}, \quad V_{(2)}=\sqrt{\operatorname{det}\left(g_{(2)} / \alpha^{\prime}\right)}
$$

are the volume of $T^{4}$ and $T^{2}$ respectively. 


\section{References}

[1] R. Blumenhagen, C. Kounnas and D. Lüst, JHEP 0001 (2000) 036.

[2] I. Antoniadis, G. D'Apollonio, E. Dudas and A. Sagnotti, Nucl. Phys. B553 (1999) 133.

[3] M. Bianchi, G. Pradisi and A. Sagnotti, Nucl. Phys. B376 (1992) 365.

[4] M. Bianchi, Nucl. Phys. B528 (1998) 73.

[5] Z. Kakushadze, G. Shiu and S.H. Henry Tye, Phys. Rev. D58 (1998) 86001;

Z. Kakushadze, hep-th/0001212.

[6] C. Angelantonj, Nucl. Phys. B566 (2000) 126.

[7] E. Kiritsis and C. Kounnas, Nucl. Phys. B503 (1997) 117.

[8] J. Scherk and J. H. Schwarz, Phys. Lett. B82 (1979) 60; Nucl. Phys. B153 (1979) 61;

E. Cremmer, J. Scherk, J. H. Schwarz, Phys. Lett. B84 (1979) 83;

C. Kounnas and B. Rostand, Nucl. Phys. B341 (1990) 641.

[9] A. Gregori, E. Kiritsis, C. Kounnas, N.A. Obers, P.M. Petropoulos and B. Pioline, Nucl. Phys. B510 (1998) 423.

[10] A. Gregori and C. Kounnas, Nucl. Phys. B560 (1999) 135;

A. Gregori, hep-th/0002214. Published on JHEP, in the proceedings of the 9'th TMR Meeting on "Quantum Aspects of Gauge Theories, Supersymmetry and Unification", Paris, France, 1-7 September 1999.

[11] M. B. Green, J. A. Harvey and G. Moore, Class. Quant. Grav 14, 1997, 47;

K. Dasgupta, D. P. Jatkar and S. Mukhi, Nucl. Phys. B523 (1998) 465.

[12] A. Sagnotti, Phys. Lett. B294 (1992) 196;

I. Antoniadis, C. Bachas, C. Fabre, H. Partouche and T. R. Taylor, Nucl. Phys. B489 (1997) 160;

I Antoniadis, H. Partouche and T. R. Taylor, Nucl. Phys. B499 (1997) 29.

[13] A. Gregori, C. Kounnas and P.M. Petropoulos, Nucl. Phys. B537 (1999) 317;

A. Gregori, talk given at the "6th Hellenic School and Workshop on Elementary Particle Physics", Corfu, Greece, 6-26 September 1998, in "Corfu 1998, Quantum aspects of gauge theories, supersymmetry and unification" 348-355.

[14] E. Kiritsis, Lectures presented at the Catholic University of Leuven and University of Padova, 1996-1997. Leuven, Belgium: Leuven Univ. Pr. (1998), Leuven notes in mathematical and theoretical physics, B9; hep-th/9709062.

[15] I. Antoniadis, E. Gava and K.S. Narain, Phys. Lett. B283 (1992) 209; Nucl. Phys. B383 (1992) 93. 
[16] J. A. Harvey and G. Moore, Nucl. Phys. B463 (1996) 315.

[17] E. Kiritsis and C. Kounnas, Nucl. Phys. B442 (1995) 472.

[18] A. Gregori, C. Kounnas and J. Rizos, Nucl. Phys. B549 (1999) 16.

[19] E. Kiritsis, C. Kounnas, P.M. Petropoulos and J. Rizos, hep-th/9605011, in the Proceedings of the 5'th Hellenic School and Workshops on Elementary Particle Physics, Corfu, Greece, 3-24 Sep. 1995; Phys. Lett. B385 (1996) 87; Nucl. Phys. B483 (1997) 141 ;

P.M. Petropoulos and J. Rizos, Phys. Lett. B374 (1996) 49.

[20] E. Kiritsis, C. Kounnas, P.M. Petropoulos and J. Rizos, Phys. Lett. B385 (1996) 87.

[21] A. Gregori, C. Kounnas and P.M. Petropoulos, Nucl. Phys. B553 (1999) 108.

[22] E. Kiritsis, C. Kounnas, P.M. Petropoulos and J. Rizos, Nucl. Phys. B540 (1999) 87.

[23] M. Serone, Phys. Lett. B395 (1997) 42, Erratum-Phys. Lett. B401 (1997) 363;

J.F. Morales and M. Serone, Nucl. Phys. B481 (1996) 389.

[24] M. Bianchi and A. Sagnotti, Nucl. Phys. B361 (1991) 519;

E. Gimon and J. Polchinski, Phys. Rev. D54 (1996) 1667.

[25] I. Antoniadis, E. Dudas and A. Sagnotti, Nucl. Phys. B544 (1999) 469.

[26] S. Chaudhuri, S.-W. Chung, G. Hockney and J. Lykken, Nucl. Phys. B456 (1995) 89; S. Chaudhuri, G. Hockney and J. D. Lykken, Phys. Rev. Lett. 75 (1995) 2267; Nucl. Phys. B469 (1996) 357.

[27] E. Witten, JHEP 9802 (1998) 006.

[28] A. Sen, Int. J. Mod. Phys. A9, 1994, 3707. 